\title{
Drug Factory Microbiome: Top Three Actionable Challenges and Prospects
}

\author{
Mona T. Kashef ${ }^{1,2}$ and Ramy K. Aziz $z^{1,2}$
}

\section{To the Editor:}

Microbiome research is advancing on diverse and multiple frontiers. An interesting and emerging arena is the microbiome of built environments and how it affects human health and lifestyle. Recently, drug factories were studied as interesting built environments in which humans, machines, and pharmaceutical ingredients interact, in a quite clean environment (which ranges from fully aseptic, in case of parenteral products, to monitored clean areas, for other types of formulations). However, studies addressing microbiomes of drug factories, clean rooms, or production lines remain scarce. Looking forward to 2020, we highlight three actionable emerging challenges in drug factory microbiome research, which might transform to prospects for clinical or discovery science innovation if they are adequately and promptly addressed.

\section{Actionable Challenge 1}

Studies on drug factory microbiomes revealed that they, being in typically protected built environments, were substantially affected by exogenous factors, such as the personnel, temperature of the production process, and the presence or absence of water (Hamdy et al., 2018; Kawai et al., 2019). Factors that are inherent to the process, such as the nature of manufactured product (i.e., whether it has antimicrobial activity), had less than expected effect (Hamdy et al., 2018); yet antimicrobial resistance genes were detected (Hamdy et al., 2018; Kawai et al., 2019). As this environment is not fully isolated, it can affect the human communities connected to it. Workers may well transfer these microbes to their contacts and outdoor environments. Therefore, the drug factory microbiome, and its resistome, may represent a reservoir for resistance genes, which may be horizontally transferred to pathogenic bacteria. Being an important yet neglected environment, the drug factory microbiome should be more systematically studied in the coming decade. Studies comparing drug factories with surrounding open and built environments, and experiments tracing workers and their microbiomes at work, home, and in commute may provide insights into the dynamics of transfer of bacteria as well as the impact of humans on shaping microbial communities.

\section{Actionable Challenge 2}

Microbiome studies can offer insights for process control improvements within the drug factory. A recent study on air and water samples from a pharmaceutical plant showed that, although the water microbiome is affected by the applied sanitization method, the air microbiome did not vary between different tested areas, but was affected by seasonal variations (Pacchioni et al., 2018). Alternative methods for air and water sanitization may guarantee proper microbiological quality of the environment, and may avoid microbiological quality crises and failures that were, until recently, of unknown cause. In the next decade, studies on the microbiome composition in relation to various disinfection and sanitization regimens may end up transforming the quality assurance and quality control processes in factories to become fully culture independent. With the availability of handheld sequencing instruments, and the continuous drop in cost, it is possible to see, in a few years, new standard operating procedures and quality control tests that merely use DNA sequencing for identification and quantification of microbes.

\section{Actionable Challenge 3}

The massive use of 16S rRNA gene profiling is allowing the exploration of microbial composition of various environments at an unprecedented pace and to an unprecedented extent, as yet-to-be cultured microbes are the major colonizers of any environment. Recently, with the development, automation, and cost reduction of RNA-sequencing approaches, it has become feasible to detect the gene expression profile in complex communities and provide data on their different functions. These metatranscriptomic studies usually complement those relying on DNA, and help in providing a full picture of the microbial composition and activity in any environment (Bashiardes et al., 2016; Marcelino et al., 2019). A grand challenge for the next decade is to apply such approaches in drug manufacturing units, as no data are yet available on microbial metatranscriptomics of these unitsto the best of our knowledge.

\section{Conclusions}

As sequencing technologies will continue to increase in speed and throughput, although the cost and instrument size decrease in the coming decade, we expect that sequencingbased technologies (microbiome profiling, metagenomics, and metatranscriptomics) will completely transform quality assurance, quality control, and epidemiological studies in clean rooms and drug manufacturing units. Further studies of

\footnotetext{
${ }^{1}$ Department of Microbiology and Immunology, Faculty of Pharmacy, Cairo University, Cairo, Egypt.

${ }^{2}$ Center for Genome and Microbiome Research, Faculty of Pharmacy, Cairo University, Cairo, Egypt.
} 
the drug factory microbiome and other built environments are essential to develop, optimize, and standardize such protocols before one can begin to decipher their impacts on pharmaceutical discovery, development, and manufacturing.

Research on microbiome of the built environments has crosscutting relevance for human and planetary health, not to mention for rational design of the future drug factories, and will remain a hot topic in the coming decades.

\section{Author Disclosure Statement}

The authors declare that no conflicting financial interests exist. M.T.K. and R.K.A. are supported by the Egyptian Academy for Scientific Research and Technology (ASRT) JESOR Grant (contract no. 41, awarded in 2018).

\section{Funding Information}

No funding was directed toward the publication of this letter.

\section{References}

Bashiardes S, Zilberman-Schapira G, and Elinav E. (2016). Use of metatranscriptomics in microbiome research. Bioinform Biol Insights 10, 19-25.

Hamdy AM, El-Massry M, Kashef MT, Amin MA, and Aziz RK. (2018). Toward the drug factory microbiome: Microbial community variations in antibiotic-producing clean rooms. OMICS 22, 133-144.

Kawai M, Ichijo T, Takahashi Y, et al. (2019). Culture independent approach reveals domination of human-oriented microbes in a pharmaceutical manufacturing facility. Eur $\mathbf{J}$ Pharm Sci 137, 104973.

Marcelino VR, Wille M, Hurt AC, et al. (2019). Metatranscriptomics reveals a diverse antibiotic resistance gene pool in avian microbiomes. BMC Biol 17, 31.

Pacchioni F, Esposito A, Giacobazzi E, Bettua C, Struffi P, and Jousson O. (2018). Air and waterborne microbiome of a pharmaceutical plant provide insights on spatiotemporal variations and community resilience after disturbance. BMC Microbiol 18, 124.

Address correspondence to: Ramy K. Aziz, PhD Department of Microbiology and Immunology Faculty of Pharmacy Cairo University Qasr El-Ainy Street

Cairo 11562 Egypt

E-mail: ramy.aziz@gmail.com 